\title{
AVALIAÇÃO DE INVESTIMENTOS EM MODERNIZAÇÃO DOS PORTOS POR ANÁLISE ENVOLTÓRIA DE DADOS
}

\author{
Luiz Octávio Gavião \\ Escola Superior de Guerra (ESG) \\ Fortaleza de São João - Av. João Luiz Alves, s/nº - Urca - Rio de Janeiro-RJ - CEP: 22291-090 \\ E-mail: luiz.gaviao67@gmail.com \\ Lidia Angulo Meza \\ Universidade Federal Fluminense (UFF) \\ Rua Passo da Pátria, 156, Bloco D, São Domingos, Niterói-RJ, CEP: 24.220-240 \\ E-mail: lidia.angulo.meza@gmail.com \\ Gilson Brito Alves Lima \\ Universidade Federal Fluminense (UFF) \\ Rua Passo da Pátria, 156, Bloco D, São Domingos, Niterói-RJ, CEP: 24.220-240 \\ E-mail: glima@id.uff.br \\ Pauli Adriano de Almada Garcia \\ Universidade Federal Fluminense (UFF) \\ Rua Des. Ellis Hermydio Figueira, 783 - Aterrado, Volta Redonda - RJ, CEP: 27213-145 \\ E-mail: pauliadriano@gmail.com \\ Sergio Kostin \\ Escola Superior de Guerra (ESG) \\ Fortaleza de São João - Av. João Luiz Alves, s/nº - Urca - Rio de Janeiro-RJ - CEP: 22291-090 \\ E-mail: sfkostin@gmail.com
}

\section{RESUMO}

As rotas marítimas desempenham um papel fundamental no comércio mundial. No Brasil, aproximadamente 95\% do comércio externo transita por redes hidroviárias. Os gargalos logísticos impõem um impacto negativo no crescimento nacional e motivam a pesquisa acadêmica e institucional. O Instituto de Pesquisa Econômica Aplicada (IPEA) e a Agência Nacional de Transportes Aquaviários (ANTAQ) desenvolveram respectivamente projetos de modernização e cenários para o aumento da movimentação de carga nos portos brasileiros até 2023. No entanto, a atual crise econômica impõe austeridade no investimento público. A Análise Envoltória de Dados (DEA) foi aplicada ao problema, a fim de identificar a eficiência do projeto e propor metas tangíveis para o investimento público. Um modelo orientado a input DEA BCC identificou nove portos eficientes e possibilidades de redução de gastos em 19 portos.

Palavra-chave: Portos brasileiros; DEA; investimentos públicos.

\begin{abstract}
Maritime routes play a key role in world trade. In Brazil, approximately 95\% of external trade transits by waterway networks. Logistic bottlenecks impose a negative impact on national growth and have motivated academic and institutional research. The Institute of Applied Economic Research (IPEA) and the National Agency of Waterway Transportation (ANTAQ) respectively developed modernization projects and scenarios for increasing cargo handling in Brazilian ports until 2023. However, the present economic crisis imposes austerity in public investment. Data Envelopment Analysis (DEA) was applied to IPEA and ANTAQ data, in order to identify project efficiency and propose tangible goals for public investment. A DEA BCC input-oriented model identified nine efficient ports and slacks that enable to decrease investment in 19 ports.
\end{abstract}

Keywords: Brazilian ports; DEA; public investment.

\section{Como Citar:}


GAVIÃO, luiz octávio; MEZA, lidia angulo; LIMA, gilson brito alves; GARCIA, pauli adriano de almada; KOSTIN, sergio. Avaliação de investimentos em modernização dos portos por Análise Envoltória de Dados. In: SIMPÓSIO DE PESQUISA OPERACIONAL E LOGÍSTICA DA MARINHA, 19., 2019, Rio de Janeiro, RJ. Anais [...]. Rio de Janeiro: Centro de Análises de Sistemas Navais, 2019. 


\section{INTRODUÇÃO}

A navegação representa o modo mais expressivo do transporte comercial de cargas em âmbito mundial. O transporte marítimo contribui com aproximadamente $80 \%$ da carga globalmente movimentada. Especificamente no Brasil, um país geograficamente favorecido por ampla malha hidrográfica de mais de 40 mil quilômetros de rios navegáveis e uma costa oceânica superior a 8 mil quilômetros, o modo marítimo transporta aproximadamente 95\% dos produtos do comércio exterior. Esses números traduzem a importância de uma adequada infraestrutura que garanta um eficiente fluxo de mercadorias, onde os portos ocupam papel de protagonistas [1].

Os "gargalos" na infraestrutura portuária se tornaram mais evidentes nas últimas décadas de intenso e crescente comércio marítimo. Filas de caminhões nas estradas que demandam os portos em períodos de safra, insuficiência das redes ferroviárias, limitações portuárias para atracação de navios de grande porte, retardo nas operações de carga e descarga, dentre outros, são alguns dos frequentes problemas. Em comparação com os demais modos rodoviário, ferroviário e aeroportuário, pesquisas comparadas em competitividade mundial apontam a infraestrutura portuária como a pior no país e a $122^{\mathrm{a}}$ classificada entre 144 países. Países vizinhos e emergentes ocupam posições melhores que o Brasil no ranking do modo aquaviário: Chile (35 $)$, África do Sul $\left(46^{\mathrm{a}}\right)$, Uruguai $\left(48^{\mathrm{a}}\right)$, China $\left(53^{\mathrm{a}}\right)$, Índia $\left(73^{\mathrm{a}}\right)$ e Rússia $\left(81^{\mathrm{a}}\right)$. Esses dados revelam a dimensão do desajuste entre a vocação marítima do país e as deficiências de infraestrutura do setor [2].

Este problema logístico de graves proporções para a economia do país tem motivado a busca de soluções ao longo da última década, com destaque aos estudos coordenados no Instituto de Pesquisa Econômica Aplicada (IPEA), denominado Mapeamento IPEA de Obras Portuárias e na Agência Nacional de Transportes Aquaviários (ANTAQ), denominado Plano Geral de Outorgas (PGO). Esses órgãos desenvolveram trabalhos com foco em duas vertentes diferentes: o primeiro aborda investimentos em 265 projetos para modernizar a infraestrutura em 31 portos brasileiros, que incluem a ampliação dos acessos por outros modais e obras em geral nos portos e nas áreas marítimas adjacentes; a segunda vertente se refere à simulação de cenários de movimentação de carga nos portos, com moldura temporal até o ano de 2023 [3]-[6].

Este artigo assume como premissas que os projetos de investimentos levantados no Mapeamento IPEA de Obras Portuárias são financeiramente exequíveis no prazo estabelecido e que os cenários de movimentação de cargas do PGO apresentam elevada probabilidade de materialização em 2023. Assim, para cada conjunto de projetos previstos para um determinado porto, tem-se um montante de recursos financeiros para obras de construção, ampliação e recuperação da infraestrutura, em paralelo com estimativas de aumento da quantidade de carga a ser movimentada, conforme a previsão dos modelos logísticos. Sob o ponto de vista do conceito de produtividade, aplicado aos portos modernizados até 2023, é possível considerar os investimentos planejados como recursos de entrada (inputs) e a capacidade ampliada dos portos como recursos de saída (output).

Nesse contexto, os portos contemplados com vultosos recursos, porém com reduzida estimativa de aumento do volume de cargas movimentadas, indicariam uma reduzida produtividade aos órgãos gestores e a consequente possibilidade de reavaliação do portfólio de projetos. Por outro lado, portos com elevada produtividade representariam uma fronteira de eficiência a ser avaliada como referência aos demais, pois apresentam melhor relação entre inputs e outputs. Dessa forma, verifica-se que a modelagem por Análise Envoltória de Dados (DEA) é adaptável ao problema de pesquisa, no caso a avaliação de 
investimentos em modernização dos portos, permitindo identificar o nível de eficiência dos diferentes portfólios, localizar a ineficiência e propor metas tangíveis para a economia de recursos [7].

A abordagem DEA orientada a inputs se torna especialmente oportuna ao problema, pois permite identificar projetos com custos excessivos, se mantidos fixos os cenários de demanda de produtos em 2023. O contexto econômico atual configura um quadro de estagnação, com possível recessão no curto prazo, impõe a necessidade de reavaliação dos custos projetos. O montante de recursos levantados pelo IPEA para a modernização dos portos poderia ser reajustado, com vistas à economia em alguns projetos modelados como ineficientes. Em resumo, a modelagem DEA indica se os investimentos levantados para uma região portuária se justificam, à luz das demandas projetadas para 2023 [7], [8].

Após os parágrafos introdutórios, a Seção 2 apresenta uma breve revisão da literatura, acerca dos estudos de modernização dos portos mencionados e da modelagem DEA, a Seção 3 descreve a metodologia utilizada ao problema, a Seção 4 traz a análise dos resultados e, por fim, a Seção 5 conclui a pesquisa.

\section{REVISÃO DA LITERATURA}

Uma breve revisão da literatura enfoca as pesquisas desencadeadas no IPEA e na ANTAQ, incluindo os principais conceitos da modelagem DEA aplicados ao problema.

\subsection{MAPEAmento IPEA de Obras Portuárias}

O Mapeamento IPEA de Obras Portuárias, desenvolvido por [5] e [6], teve por finalidade dimensionar e avaliar demandas, deficiências e "gargalos" dos portos brasileiros. O estudo descreve a deficiência de infraestrutura, que compromete o potencial do setor e provoca um entrave ao crescimento do comércio internacional e de cabotagem no Brasil. $\mathrm{O}$ estudo seguiu dois critérios: (1) identificação de projetos documentados em planos e programas do setor público e privado; (2) ponderação destes documentos para selecionar o valor de cada projeto, com prioridade às obras previstas no Programa de Aceleração do Crescimento (PAC), ao Plano Plurianual (PPA) 2008-2012 e ao Plano Nacional de Logística e Transportes-PNLT [9]-[11], nessa ordem.

O estudo quantificou os investimentos necessários no setor, a partir de um levantamento de 265 obras de infraestrutura portuária, de acesso ou de apoio. As diversas obras abrangem 133 projetos de construção, ampliação e recuperação de áreas nos portos (R \$ 20,46 bilhões), 45 projetos nos acessos terrestres aos portos (R \$ 17,29 bilhões), 46 projetos de dragagem e derrocamento (R $\$ 2,78$ bilhões) e 41 projetos de infraestrutura portuária (R\$2,34 bilhões) [5], [6].

Embora o IPEA não tenha publicado uma atualização aos recursos planejados no PAC 2, com vigência entre 2011 e 2014, a diferença entre os "gargalos" identificados e os investimentos orçados no PAC 1 (2007-2010) é significativa. Das 265 obras levantadas no estudo do IPEA, apenas 51 constavam no PAC 1 . Isto representava 19,2\% do número de obras necessárias, segundo o IPEA, correspondendo a 23\% do total orçado. Dentre as obras mais atendidas estão as dragagens, atendendo a 55,3\% das deficiências identificadas, e as obras em acessos terrestres aos portos, com 39,2\%. Considerando que diversas obras enfrentam problemas com atrasos, somados às necessidades de contingenciamentos decorrentes da atual conjuntura econômica, dentre outros problemas jurídicos que envolvem as maiores empreiteiras do país, não se estima que uma atualização de dados ao PAC 2 alteraria de forma considerável as divergências com o Mapeamento IPEA de Obras Portuárias [12]. 


\subsection{Plano Geral de Outorgas Portuárias}

Em extensão as suas atividades como agência reguladora e fiscalizadora do setor de transportes aquaviários, a ANTAQ elaborou o PGO, com caráter orientador ao planejamento portuário, contribuindo ao fomento do crescimento do setor. O PGO decorreu de uma resolução normativa, no caso a Lei no 10.233/2001 e, posteriormente, do Decreto no $6.620 / 2008$, de forma a garantir que os investimentos e a outorga governamental para projetos portuários se mantivessem alinhados às diretrizes da política de transportes do setor [3], [4].

Com base no PNLT, o PGO incluiu cenários de investimentos até 2023, a partir da identificação de áreas com potencial para receber novos portos ou ampliar os existentes. Essas áreas foram levantadas a partir de análise em três etapas técnicas: primeiro, a projeção das cargas a serem escoadas por cada vetor logístico; segundo, a profundidade mínima dos portos com as cargas esperadas e, por fim, as malhas rodoviária, ferroviária e hidroviária, implantadas ou projetadas, próximas às áreas indicadas para os portos [3], [4].

O processo de modelagem da estimativa de demandas para os portos se iniciou com a definição da área de influência de estudo, seu zoneamento e a preparação da rede de transportes, que representa a infraestrutura existente e os projetos em desenvolvimento. Em seguida, a pesquisa se desenvolveu em quatro etapas distintas: geração de viagens ou da demanda; distribuição de viagens ou da demanda; divisão ou escolha do modo; e alocação das viagens às redes de transportes. A agregação dos produtos de maior expressão movimentados nos portos foi associada a cinco grupos para facilitar a análise: granéis vegetais, granéis líquidos, granéis minerais, carga geral e contêineres.

A Tabela 1 reúne a pesquisa do IPEA e as orientações da ANTAQ, sintetizando os montantes de recursos planejados no Mapeamento IPEA de Obras Portuárias e os totais de cargas movimentadas nos portos, por categorias, levantadas no PGO, com os dados efetivos do momento inicial da pesquisa, em 2007 e os dados projetados por modelagem logística para 2023. A Tabela 1 apresenta a base de dados inicial desta pesquisa.

Tabela 1: síntese dos trabalhos do IPEA e ANTAQ

\begin{tabular}{|c|c|c|c|c|c|c|c|c|c|c|c|c|}
\hline \multirow[t]{2}{*}{ Portos } & \multirow[t]{2}{*}{ UF } & \multirow[t]{2}{*}{$\begin{array}{l}\text { Mapa IPEA } \\
\text { (R\$ milhões) }\end{array}$} & \multicolumn{2}{|c|}{$\begin{array}{c}\text { Granéis } \\
\text { Vegetais } \\
\text { (mil toneladas) }\end{array}$} & \multicolumn{2}{|c|}{$\begin{array}{c}\text { Granéis } \\
\text { Minerais } \\
\text { (mil toneladas) }\end{array}$} & \multicolumn{2}{|c|}{$\begin{array}{l}\text { Granéis } \\
\text { Líquidos } \\
\text { (mil ton) }\end{array}$} & \multicolumn{2}{|c|}{$\begin{array}{l}\text { Carga Geral } \\
\text { (mil toneladas) }\end{array}$} & \multicolumn{2}{|c|}{$\begin{array}{l}\text { Contêineres } \\
\text { (mil TEUs) }\end{array}$} \\
\hline & & & 2007 & 2023 & 2007 & 2023 & 2007 & 2023 & 2007 & 2023 & 2007 & 2023 \\
\hline Angra dos Reis & $\mathrm{RJ}$ & 255,2 & 0 & 0 & 0 & 0 & 448 & 784 & 0 & 0 & 0 & 0 \\
\hline Antonina & PR & 27,4 & 0 & 0 & 13 & 24 & 0 & 0 & 0 & 0 & 0 & 0 \\
\hline Aratu & $\mathrm{BA}$ & 659,59 & 253 & 316 & 2620 & 4582 & 6798 & 11892 & 175 & 300 & 0 & 0 \\
\hline Belém & PA & 35,2 & 165 & 206 & 99 & 173 & 354 & 619 & 107 & 200 & 70 & 170 \\
\hline Cabedelo & PB & 372,2 & 62 & 78 & 214 & 374 & 0 & 0 & 75 & 150 & 0 & 0 \\
\hline Fortaleza & $\mathrm{CE}$ & 537,8 & 788 & 984 & 151 & 265 & 271 & 473 & 409 & 700 & 64 & 180 \\
\hline Ilhéus & $\mathrm{BA}$ & 2730,56 & 0 & 0 & 11 & 19 & 0 & 0 & 0 & 0 & 0 & 0 \\
\hline Imbituba & SC & 1932,13 & 107 & 134 & 585 & 1024 & 110 & 193 & 38 & 50 & 15 & 40 \\
\hline Itaguaí & $\mathrm{RJ}$ & 2211,68 & 0 & 0 & 4350 & 7610 & 0 & 0 & 1431 & 2500 & 227 & 0 \\
\hline Itajaí & SC & 514,49 & 0 & 0 & 0 & 0 & 67 & 117 & 133 & 250 & 682 & 1700 \\
\hline Itaqui & MA & 1473,48 & 71 & 89 & 6026 & 10541 & 197 & 345 & 4124 & 7200 & 0 & 200 \\
\hline Maceió & $\mathrm{AL}$ & 569,37 & 76 & 95 & 88 & 154 & 1021 & 1786 & 404 & 700 & 6 & 20 \\
\hline Manaus & $\mathrm{AM}$ & 270,2 & 0 & 0 & 48 & 83 & 2030 & 3551 & 206 & 350 & 176 & 510 \\
\hline Natal & $\mathrm{RN}$ & 140,5 & 76 & 95 & 25 & 43 & 29 & 51 & 6 & 10 & 8 & 20 \\
\hline
\end{tabular}




\begin{tabular}{|c|c|c|c|c|c|c|c|c|c|c|c|c|}
\hline \multirow[t]{2}{*}{ Portos } & \multirow[t]{2}{*}{ UF } & \multirow[t]{2}{*}{$\begin{array}{l}\text { Mapa IPEA } \\
\text { (R\$ milhões) }\end{array}$} & \multicolumn{2}{|c|}{$\begin{array}{c}\text { Granéis } \\
\text { Vegetais } \\
\text { (mil toneladas) }\end{array}$} & \multicolumn{2}{|c|}{$\begin{array}{c}\text { Granéis } \\
\text { Minerais } \\
\text { (mil toneladas) }\end{array}$} & \multicolumn{2}{|c|}{$\begin{array}{l}\text { Granéis } \\
\text { Líquidos } \\
\text { (mil ton) }\end{array}$} & \multicolumn{2}{|c|}{$\begin{array}{l}\text { Carga Geral } \\
\text { (mil toneladas) }\end{array}$} & \multicolumn{2}{|c|}{$\begin{array}{l}\text { Contêineres } \\
\text { (mil TEUs) }\end{array}$} \\
\hline & & & 2007 & 2023 & 2007 & 2023 & 2007 & 2023 & 2007 & 2023 & 2007 & 2023 \\
\hline Paranaguá & PR & 2178 & 207 & 259 & 170 & 298 & 1790 & 3131 & 669 & 1150 & 595 & 1460 \\
\hline Pecém & $\mathrm{CE}$ & 2683,065 & 0 & 0 & 0 & 0 & 0 & 0 & 0 & 0 & 144 & 380 \\
\hline Porto Alegre & RS & 100,2 & 80 & 99 & 14 & 25 & 3126 & 5469 & 26 & 50 & 16 & 50 \\
\hline Recife & $\mathrm{PE}$ & 68,3 & 505 & 631 & 683 & 1194 & & & 285 & 500 & 0 & 0 \\
\hline Rio de Janeiro & $\mathrm{RJ}$ & 1893,84 & 796 & 994 & 1070 & 1890 & 766 & 1340 & 3398 & 5950 & 390 & 980 \\
\hline Rio Grande & RS & 1085,6 & 1762 & 2912 & 502 & 878 & 2378 & 4161 & 1630 & 5600 & 575 & 1810 \\
\hline Salvador & BA & 677,04 & 413 & 516 & 9 & 16 & 0 & 0 & 128 & 200 & 230 & 610 \\
\hline Santarém & PA & 60,15 & 0 & 0 & 84 & 148 & 0 & 0 & 0 & 0 & 0 & 10 \\
\hline Santos & SP & 8670,31 & 1615 & 2017 & 6291 & 11006 & 7120 & 12456 & 6206 & 13900 & 2525 & 8870 \\
\hline $\begin{array}{l}\text { São Francisco do } \\
\text { Sul }\end{array}$ & SC & 1360,21 & 285 & 356 & 143 & 250 & 201 & 352 & 117 & 200 & 244 & 610 \\
\hline São Sebastião & SP & 156,25 & 0 & 0 & 462 & 808 & 2245 & 3927 & 25 & 50 & 0 & 0 \\
\hline Suape & $\mathrm{PE}$ & 2814,765 & 0 & 0 & 182 & 319 & 493 & 862 & 148 & 250 & 195 & 1070 \\
\hline Vila do Conde & PA & 348,2 & 0 & 0 & 5262 & 9205 & 1485 & 2598 & 0 & 0 & 31 & 90 \\
\hline Vitória & ES & 2729,77 & 404 & 504 & 13462 & 23552 & 165 & 289 & 11958 & 20900 & 266 & 690 \\
\hline
\end{tabular}

Fonte: adaptado de Campos Neto et al. (2009a) e ANTAQ (2009a).

Na Tabela 1, a coluna "Portos" inclui somente as instalações listadas nas pesquisas do IPEA e da ANTAQ. Assim, foram excluídos os portos de Santana-PA e o Terminal Inácio Barbosa-SE, não incluídos no Mapeamento IPEA de Obras Portuárias e os portos que não citados no PGO: Alcântara-MA, Areia Branca-RN, Barra do Riacho-ES, Barra GrandeCE, Centro Amazônico-AM, Espadarte-PA, Itacoatiara-AM, Juazeiro-BA, Laguna-SC, Mercosul-PR, Niterói-RJ, Norte Fluminense-RJ, Novo Rio Grande-RS, Petrolina-PE, Pontal do Sul-PR, Porto Velho-RO, Praia Mole-ES, Santa Vitória do Palmar-RS, São Luís-MA, São Simão-GO, Sotave-PA, Terminal Norte Capixaba-ES, Terminais Hidroviários-AM, Tubarão-ES e Ubu-ES.

A coluna "Mapa IPEA" soma os recursos orçados por [5] e [6] para as diferentes obras de infraestrutura, dragagem, nos acessos terrestres, dentre outras, para cada porto. As colunas de diferentes granéis são mensuradas em unidades de mil toneladas, a partir dos dados do PGO, com medidas efetivas de 2007 e projeções para 2023. A coluna "Contêineres" utiliza a unidade padronizada equivalente ao contêiner de 20 pés de comprimento, denominada Twenty-foot Equivalent Unit (TEU), também com medidas efetivas de 2007 e projeções para 2023.

\subsection{MODELAGEM DEA}

DEA é uma metodologia não paramétrica com base em programação linear, sendo usada para medir a eficiência de unidades produtivas, dentre outras utilidades. Considerando que as unidades produtivas tomam decisões com a finalidade de melhor utilizarem seus recursos no processo de produção, em DEA tais unidades assumem a denominação de Unidades Tomadoras de Decisão (no idioma inglês, Decision Making Units-DMU).

Em DEA, eficácia, produtividade e eficiência são conceitos específicos e relevantes. Segundo [7], a eficácia de uma DMU se relaciona à quantidade produzida, sem considerar os recursos utilizados na produção; a produtividade de uma DMU significa a razão entre produtos (outputs) e recursos (inputs) de cada DMU; e a eficiência é uma medida de comparação da produtividade entre as DMU. Dessa forma, o método compara o que foi 
produzido, dado os recursos disponíveis, com o que poderia ter sido produzido com os mesmos recursos.

DEA também é usada para benchmarking em gestão de operações, onde um conjunto de medidas é selecionado para aferir o desempenho da produção e de serviços, estabelecendo fronteiras de "melhores práticas". Sob esse ponto de vista, as diferentes características (i.e indicadores, resultados, métricas, outros) componentes do sistema são classificadas como inputs e outputs, para permitir a aplicação do método DEA [8].

O método DEA pode ser analisado por diferentes modelos, com destaque para as versões consideradas “clássicas”, desenvolvidos por [13], denominado CCR e por [14], denominado BCC. O modelo CCR se baseia no axioma da proporcionalidade em relação aos incrementos em inputs e outputs, sendo assim caracterizado como um modelo de retornos constantes de escala (CRS). O modelo BCC se baseia no axioma da convexidade, onde incrementos nos inputs podem representar retornos decrescentes, proporcionais ou crescentes aos outputs, sendo assim também designado como um modelo de retornos variáveis de escala (VRS).

Os modelos CCR e BCC podem ser orientados para a redução dos inputs ou ampliação dos outputs, dependendo das necessidades do decisor e/ou da natureza do problema. Quando orientados a inputs, o modelo matemático torna constantes os produtos e busca reduzir os recursos. Para tal, presume-se que o decisor controla as variáveis de entrada e existe coerência racional para a redução dos inputs. Para ilustrar esse aspecto, para um input "número de funcionários", a sua redução poderia significar a demissão de mão-deobra, gerando uma situação socialmente indesejável ou mesmo uma situação irreal, no caso de impossibilidade de demissão de funcionários. A decisão pela orientação do problema DEA requer minuciosa análise do decisor, segundo [7].

\subsection{APLICAÇÕES DEA EM PORTOS}

O uso de modelos em DEA para a análise de eficiência em portos tem sido crescente nos últimos anos. O Quadro 1 apresenta os principais artigos entre 2017 e 2019, que trazem aplicações DEA em portos. As pesquisas envolvem portos em diferentes países e o emprego de diversos modelos DEA. 
Quadro 1 - Aplicações DEA em portos

\begin{tabular}{|c|c|c|c|c|}
\hline $\begin{array}{l}\text { Ano / } \\
\text { Referência }\end{array}$ & Título & Periódico & Modelo DEA & Comentários \\
\hline 2019 [15] & $\begin{array}{l}\text { Benchmarking environmental } \\
\text { efficiency of ports using data } \\
\text { mining and RDEA: the case of } \\
\text { a US container ports }\end{array}$ & $\begin{array}{l}\text { International Journal of } \\
\text { Logistics Research and } \\
\text { Applications }\end{array}$ & $\begin{array}{l}\text { Mapa de Auto- } \\
\text { Organização de } \\
\text { Kohonen (KSOM) e } \\
\text { DEA Recursivo } \\
\text { (RDEA) }\end{array}$ & $\begin{array}{l}\text { O artigo apresenta práticas de benchmarking para a melhoria do desempenho ambiental } \\
\text { de portos. Uma amostra de } 20 \text { portos de contêineres nos EUA foi selecionada. Para } \\
\text { variáveis de entrada foram considerados: número de guindaste de cais, acres, berço e } \\
\text { profundidade. Para variáveis de saída foram considerados: número de chamadas, vazão } \\
\text { e tonelagem de peso morto e emissões de CO2. Entre as amostras selecionadas, oito } \\
\text { portas de contêineres são consideradas ambientalmente ineficientes. }\end{array}$ \\
\hline 2019 [16] & $\begin{array}{l}\text { Measuring West-Africa Ports } \\
\text { Efficiency Using Data } \\
\text { Envelopment Analysis }\end{array}$ & $\begin{array}{l}\text { Journal of } \\
\text { Transportation } \\
\text { Technologies }\end{array}$ & $\begin{array}{l}\text { DEA CCR, BCC e } \\
\text { Windows I-C }\end{array}$ & $\begin{array}{l}\text { A pesquisa mediu a eficiência relativa de cinco grandes portos comerciais na África } \\
\text { Ocidental, usando três modelos DEA (CCR, BCC e Windows I-C), entre 2005-2016. } \\
\text { Sete variáveis de entrada e uma variável de saída foram usadas na análise do modelo. } \\
\text { Os métodos CCR e BCC foram usados para avaliar a eficiência técnica e de escala, } \\
\text { enquanto o método Windows I-C forneceu uma classificação abrangente das portas } \\
\text { estudadas. Os resultados mostraram que o escore de eficiência da escala de 89,53\% } \\
\text { indicou que, em média, a escala de produção dos portos se desviou do tamanho da } \\
\text { escala mais produtiva (MPSS) em 10,47\%. Esses resultados revelaram que a fonte da } \\
\text { ineficiência geral é devida à escala e não à pura ineficiência técnica. }\end{array}$ \\
\hline 2019 [17] & $\begin{array}{l}\text { Avaliação da eficiência da } \\
\text { gestão ambiental e eficiência } \\
\text { operacional de portos públicos } \\
\text { brasileiros que exportam soja }\end{array}$ & $\begin{array}{l}\text { Revista de } \\
\text { Administração Pública }\end{array}$ & DEA CCR e BCC & $\begin{array}{l}\text { Sete portos públicos brasileiros que movimentam soja foram avaliados quanto a sua } \\
\text { gestão ambiental com DEA. Para a análise, os dados referentes ao gerenciamento de } \\
\text { efluentes líquidos, resíduos sólidos, e presença de fauna sinantrópica nesses portos } \\
\text { foram utilizados em conjunto com o Índice de Desempenho Ambiental da ANTAQ. Os } \\
\text { resultados mostram que a qualidade da gestão ambiental precisa ser abordada para que } \\
\text { o sistema seja eficiente como um todo. }\end{array}$ \\
\hline 2019 [18] & $\begin{array}{l}\text { Evaluating the Environmental } \\
\text { Performance and Operational } \\
\text { Efficiency of Container Ports: } \\
\text { An Application to the Maritime } \\
\text { Silk Road }\end{array}$ & $\begin{array}{l}\text { International Journal of } \\
\text { Environmental } \\
\text { Research and Public } \\
\text { Health }\end{array}$ & $\begin{array}{l}\text { Modelo Baseado em } \\
\text { Folgas (SBM) }\end{array}$ & $\begin{array}{l}\text { O desempenho ambiental de um porto de contêineres é importante para sua } \\
\text { competitividade e desenvolvimento sustentável. No entanto, os portos de contêineres } \\
\text { ao longo da Maritime Silk Road (MSR) causaram inúmeros problemas com o rápido } \\
\text { desenvolvimento, entre os quais a poluição ambiental. Os dez principais portos de } \\
\text { contêineres ao longo da MSR, incluindo os portos de Xangai, Hong Kong, Cingapura, } \\
\text { Kelang, Laem Chabang, Colombo, Dubai, Barcelona, Antuérpia e Hamburgo foram } \\
\text { mensurados em relação ao desempenho operacional e ambiental. }\end{array}$ \\
\hline 2018 [19] & $\begin{array}{l}\text { Efficiency, productivity and } \\
\text { returns to scale in ports: a } \\
\text { comparison of data } \\
\text { envelopment analysis and } \\
\text { econometric estimation with } \\
\text { application to Caribbean Small } \\
\text { Island Developing States }\end{array}$ & $\begin{array}{l}\text { Maritime Economics \& } \\
\text { Logistics }\end{array}$ & $\begin{array}{l}\text { DEA com método dos } \\
\text { mínimos quadrados e } \\
\text { Îndice de } \\
\text { Produtividade de } \\
\text { Malmquist }\end{array}$ & $\begin{array}{l}\text { Este trabalho investiga a eficiência técnica evolucionária e a produtividade de portos } \\
\text { em Pequenos Estados Insulares em Desenvolvimento (SIDS). O foco é sobre os SIDS } \\
\text { caribenhos, comparados em dois grupos: os principais portos e em outros SIDS nos } \\
\text { oceanos Pacífico e Índico. Os resultados revelam um nível significativamente mais } \\
\text { baixo de eficiência técnica no Caribe, quando avaliado em comparação com os } \\
\text { principais portos, porém maior quando comparado a outros SIDS. Os portos caribenhos } \\
\text { alcançaram uma taxa mais alta de melhoria de produtividade em comparação com os } \\
\text { principais portos e, portanto, fecharam parcialmente a lacuna de eficiência, mas não } \\
\text { tanto quanto os portos em outros SIDS. }\end{array}$ \\
\hline
\end{tabular}




\begin{tabular}{|c|c|c|c|c|}
\hline $\begin{array}{l}\text { Ano / } \\
\text { Referência }\end{array}$ & Título & Periódico & Modelo DEA & Comentários \\
\hline 2018 [20] & $\begin{array}{l}\text { Benchmarking freight } \\
\text { transportation corridors and } \\
\text { routes with data envelopment } \\
\text { analysis (DEA) }\end{array}$ & $\begin{array}{l}\text { Benchmarking: An } \\
\text { International Journal }\end{array}$ & $\begin{array}{l}\text { Análise de } \\
\text { Componentes } \\
\text { Principais (PCA) }\end{array}$ & $\begin{array}{l}\text { O objetivo da pesquisa é medir e comparar coletivamente a eficiência dos corredores } \\
\text { brasileiros e norte-americanos de transporte de soja, desde fazendeiros até portos de } \\
\text { exportação, utilizando DEA. Este trabalho utilizou medidas e variáveis baseadas em } \\
\text { folga que representam os três pilares da sustentabilidade (econômica, social e } \\
\text { ambiental). A escolha das variáveis foi orientada pela revisão da literatura e analisada } \\
\text { por meio da análise de componentes principais. Os resultados são coerentes com o } \\
\text { transporte de soja nos dois países (Brasil e EUA). Rotas e corredores eficientes tendem } \\
\text { a apresentar viagens de caminhão de curta distância e viagens de trem ou barcaças de } \\
\text { longa distância. A eficiência das viagens de navegação interior depende de quantas } \\
\text { barcaças são usadas na mesma expedição. Rotas com mais de três modos tendem a ser } \\
\text { ineficientes, o que sugere que há um limite para a multimodalidade. }\end{array}$ \\
\hline 2018 [21] & $\begin{array}{l}\text { Sustainability and interactivity } \\
\text { between cities and ports: a two- } \\
\text { stage data envelopment } \\
\text { analysis (DEA) approach }\end{array}$ & $\begin{array}{l}\text { Maritime Policy \& } \\
\text { Management }\end{array}$ & Modelos DEA SBM & $\begin{array}{l}\text { Portos e cidades estão intrinsecamente ligados. A cidade portuária, que serve de elo } \\
\text { entre a economia local e global, é uma integração dos sistemas urbano e portuário. } \\
\text { Usando DEA em dois estágios, este artigo propõe um novo método para medir o } \\
\text { desenvolvimento sustentável de diferentes sistemas de cidades portuárias. Vinte } \\
\text { principais cidades portuárias de contêineres são selecionadas para este estudo usando } \\
\text { dados contemporâneos. Os resultados mostram suas diferenças relativas nos níveis de } \\
\text { desenvolvimento sustentável, que revelam a eficácia das políticas prevalecentes neles. }\end{array}$ \\
\hline 2017 [22] & $\begin{array}{l}\text { Analysis of the efficiency of } \\
\text { port container terminals with } \\
\text { the use of the data envelopment } \\
\text { analysis method of relative } \\
\text { productivity evaluation }\end{array}$ & $\begin{array}{l}\text { Management Systems in } \\
\text { Production Engineering }\end{array}$ & DEA CCR & $\begin{array}{l}\text { O artigo apresenta um método para avaliar a eficiência dos terminais de contêineres } \\
\text { portuários. A pesquisa foi realizada para nove terminais europeus que utilizam } \\
\text { diferentes tecnologias de manipulação. Os terminais foram divididos devido ao nível } \\
\text { de automação de processos de transporte. }\end{array}$ \\
\hline 2017 [23] & $\begin{array}{l}\text { Intermodal terminal planning } \\
\text { by petri nets and data } \\
\text { envelopment analysis }\end{array}$ & $\begin{array}{l}\text { Control Engineering } \\
\text { Practice }\end{array}$ & $\begin{array}{l}\text { DEA e Redes de Petri } \\
\text { Temporizadas (TPN) }\end{array}$ & $\begin{array}{l}\text { O planejamento e a gestão de recursos em terminais intermodais é integrado em Redes } \\
\text { de Petri Temporizadas (TPN) e DEA e consiste em três etapas: a modelagem do } \\
\text { terminal via TPN para modelar o comportamento regular; a avaliação de se a } \\
\text { configuração atual pode lidar com o aumento dos fluxos de carga; se não, a análise por } \\
\text { eficiência cruzada DEA de soluções alternativas de planejamento. O procedimento } \\
\text { fornece ao tomador de decisões número, capacidade e cronograma de recursos para } \\
\text { lidar com o aumento dos fluxos. O método é avaliado por um estudo de caso real, } \\
\text { mostrando que a integração de NPT e DEA permite tomar decisões de planejamento } \\
\text { sob requisitos conflitantes. }\end{array}$ \\
\hline 2017 [24] & $\begin{array}{l}\text { Efficiency of inland waterway } \\
\text { container terminals: Stochastic } \\
\text { frontier and data envelopment } \\
\text { analysis to analyze the capacity } \\
\text { design-and throughput } \\
\text { efficiency }\end{array}$ & $\begin{array}{l}\text { Transportation } \\
\text { Research Part A: } \\
\text { Policy and Practice }\end{array}$ & $\begin{array}{l}\text { DEA e Análise de } \\
\text { Fronteiras } \\
\text { Estocásticas (SFA) }\end{array}$ & $\begin{array}{l}\text { Este trabalho se concentra na análise de eficiência dos terminais de contêineres } \\
\text { fluviais. Existem diferenças importantes entre os terminais fluviais e os marítimos em } \\
\text { termos de capacidade de projeto e, portanto, também em operações. Diferentes } \\
\text { combinações de entradas e saídas foram testadas com as metodologias SFA e DEA. } \\
\text { Importantes entradas de terminal são o pátio e o guindaste, mas também as horas de } \\
\text { operação do terminal e a área do terminal. }\end{array}$ \\
\hline
\end{tabular}




\begin{tabular}{|c|c|c|c|c|}
\hline $\begin{array}{l}\text { Ano / } \\
\text { Referência } \\
\end{array}$ & Título & Periódico & Modelo DEA & Comentários \\
\hline 2017 [25] & $\begin{array}{l}\text { Assessment of differences in } \\
\text { efficiency across strategic } \\
\text { groups in the container } \\
\text { shipping context: a data } \\
\text { envelopment analysis }\end{array}$ & $\begin{array}{l}\text { International Journal of } \\
\text { Shipping and Transport } \\
\text { Logistics }\end{array}$ & DEA CCR e BCC & $\begin{array}{l}\text { A pesquisa examina as diferenças de eficiência entre os grupos estratégicos no } \\
\text { contexto do transporte de contêineres, usando a análise por envoltória de dados } \\
\text { secundários coletados de } 17 \text { transportadores de contêineres globais. As transportadoras } \\
\text { globais de contêineres foram classificadas com base em variáveis estratégicas como } \\
\text { capacidade da frota, índice de ativos / dívida, índice de frota própria / fretada, índice de } \\
\text { receita não-contêiner e receita. Foram identificados quatro grupos estratégicos: } \\
\text { proativo-prudente, proativo, oportunista, conservador-prudente e conservador. Os } \\
\text { resultados do modelo DEA indicaram diferenças significativas na eficiência entre os } \\
\text { quatro grupos estratégicos. }\end{array}$ \\
\hline 2017 [26] & $\begin{array}{l}\text { Grain intermodal terminals: } \\
\text { evaluation of pure technical } \\
\text { efficiency by Data Envelopment } \\
\text { Analysis }\end{array}$ & Production & DEA CCR e BCC & $\begin{array}{l}\text { Este trabalho teve como objetivo verificar se os terminais com eficiência produtiva } \\
\text { também possuem eficiência técnica pura, utilizando a técnica DEA. Os dados foram } \\
\text { coletados por meio de entrevista, utilizando questionário estruturado e não } \\
\text { probabilístico para amostragem por conveniência. Enquanto o modelo CCR identificou } \\
\text { que apenas três das doze DMU analisadas têm eficiência técnica total, através do } \\
\text { modelo BCC foi possível observar que esses terminais eram os únicos com eficiência } \\
\text { técnica pura. }\end{array}$ \\
\hline 2017 [27] & $\begin{array}{l}\text { Data envelopment analysis of } \\
\text { AGV fleet sizing at a port } \\
\text { container terminal }\end{array}$ & $\begin{array}{l}\text { International Journal of } \\
\text { Production Research }\end{array}$ & DEA CCR & $\begin{array}{l}\text { A pesquisa aplicou DEA para determinar os processos eficientes de movimentação de } \\
\text { contêineres (considerando o número de Veículos Guiados Automatizados (AGVs)) em } \\
\text { um terminal de contêineres (PCT). O processo de manuseio de contêineres, com um } \\
\text { número fixo de guindastes de cais, quando um número diferente de AGVs é usado para } \\
\text { transportar contêineres de cais para locais atribuídos dentro da área de armazenamento, } \\
\text { representa uma unidade de tomada de decisão (DMU). O modelo CCR usou dois } \\
\text { inputs: custos médios de operação e custos operacionais médios dos equipamentos } \\
\text { empregados em um PCT e dois outputs: número médio de contêineres de importação } \\
\text { por navio e utilização média ponderada taxa de equipamento em um PCT. }\end{array}$ \\
\hline 2017 [28] & $\begin{array}{l}\text { Relative efficiencies of ASEAN } \\
\text { container ports based on data } \\
\text { envelopment analysis }\end{array}$ & $\begin{array}{l}\text { The Asian Journal of } \\
\text { Shipping and Logistics }\end{array}$ & DEA Supereficiëncia & $\begin{array}{l}\text { Este estudo analisa as eficiências relativas de } 50 \text { portos e terminais de contêineres da } \\
\text { ASEAN. Estes portos são categorizados de acordo com o seu sistema e localização de } \\
\text { movimentação de contêineres. Os portos localizados em uma margem do rio foram } \\
\text { designados de "portos interiores" e os portos à beira-mar de "portos marítimos". }\end{array}$ \\
\hline
\end{tabular}




\section{METODOLOGIA}

A modelagem por DEA deve atender basicamente a três etapas para implementar o problema, segundo [29]: a definição e seleção das DMU; a seleção das variáveis; e a escolha e aplicação do modelo. Tendo em vista uma peculiaridade da base de dados ao problema desta pesquisa, referente a valores negativos e nulos em algumas variáveis, incluiu-se uma etapa adicional para o tratamento dos dados para esse caso, conforme descrito em [16] e [17].

\subsection{DEFINIÇÃO E SELEÇÃO DE DMU}

DEA Em relação à definição das DMU, o conjunto adotado deve utilizar os mesmos inputs e outputs, variando apenas em intensidade. Deve atender ao requisito de homogeneidade, realizando as mesmas tarefas, com os mesmos objetivos, submeter-se às mesmas condições de mercado e ter autonomia na tomada de decisões, conforme destaca [29].

As 28 instalações portuárias listadas na Tabela 1 atendem às características de DMU, variando em intensidade seus inputs e outputs e apresentando as condições de homogeneidade listadas, mesmo que operem sob diferentes amplitudes de escala.

\subsection{SELEÇÃO DAS VARIÁVEIS}

Os recursos previstos pelo IPEA para investimentos nos portos foram associados a inputs, enquanto os incrementos na movimentação das diferentes cargas nos portos até 2023 foram associados a outputs. Essa abordagem DEA se torna pertinente aos estudos do IPEA e ANTAQ, e oportuna ao momento do país. Uma série de necessidades na infraestrutura de transportes, conforme indicam os PAC 1 e 2, podem requerer uma reavaliação, com vistas à otimização de recursos financeiros.

As diferentes unidades de medida de movimentação de carga descritas na Tabela 1, em "mil toneladas" para cargas e "mil TEU" para contêineres, indicam a necessidade de considerar, ao menos, dois outputs diferentes. A opção de reunir as quatro categorias de cargas medidas em "mil toneladas" sob o mesmo output reflete melhor a relação entre input e outputs, pois os investimentos nos portos interferem de forma global sobre as diferentes cargas. Por exemplo, uma obra nos acessos terrestres ao porto beneficia o incremento na movimentação de carga nas diversas categorias. Assim, o problema DEA foi equacionado com 28 DMU, um input e dois outputs, conforme a Tabela 2. Os valores não-positivos foram posteriormente submetidos a tratamento, por necessidade da modelagem DEA.

Tabela 2: seleção das variáveis 


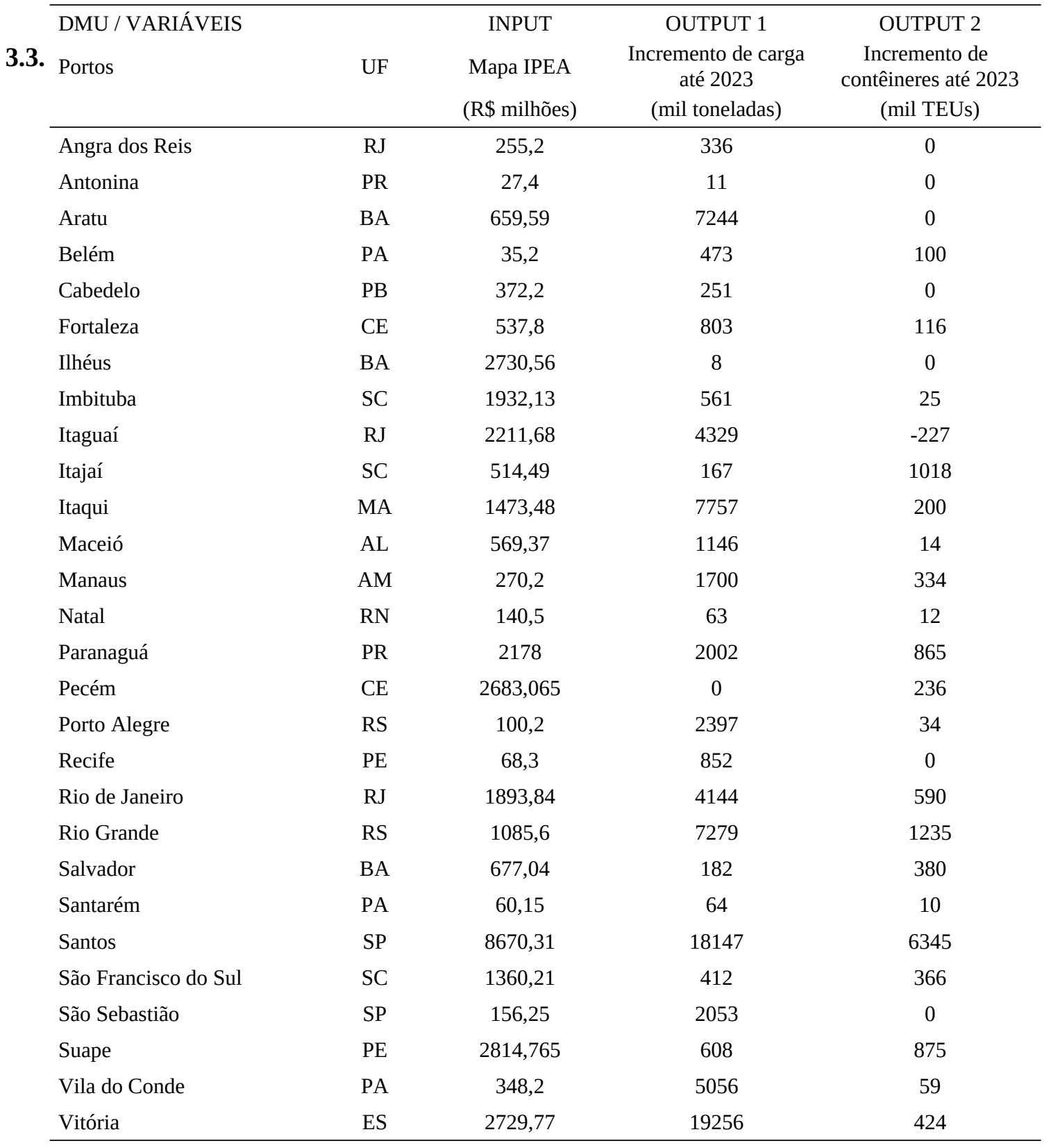

\section{TRATAMENTO DOS DADOS EM DEA}

Por vezes, o conjunto de dados pode apresentar valores negativos ou nulos, para atendimento da característica de "positividade" em DEA. Um dos métodos mais comuns para eliminar os problemas de valores não-positivos em DEA tem sido através da adição de uma constante positiva, suficientemente grande, para os valores de input ou output que incluam tais valores indesejados [31].

A Tabela 3 apresenta o tratamento dos dados, a partir do acréscimo de cem mil unidades aos outputs 1 e 2. Esse tratamento é denominado “translação”, segundo [7].

Tabela 3: tratamento dos dados

\begin{tabular}{|c|c|c|c|c|c|}
\hline \multirow[t]{2}{*}{ DMU } & VARIÁVEIS & & INPUT & OUTPUT 1 & OUTPUT 2 \\
\hline & Portos & UF & $\begin{array}{l}\text { Mapa IPEA } \\
\text { (R\$ milhões) }\end{array}$ & $\begin{array}{c}\text { Incremento de carga } \\
\text { até } 2023 \\
\text { (com translação) }\end{array}$ & $\begin{array}{l}\text { Incremento de } \\
\text { contêineres até } 2023 \\
\text { (com translação) }\end{array}$ \\
\hline
\end{tabular}




\begin{tabular}{|c|c|c|c|c|c|}
\hline DMU 1 & Angra dos Reis & $\mathrm{RJ}$ & 255,2 & 100336 & 100000 \\
\hline DMU 2 & Antonina & PR & 27,4 & 100011 & 100000 \\
\hline DMU 3 & Aratu & BA & 659,59 & 107244 & 100000 \\
\hline DMU 4 & Belém & PA & 35,2 & 100473 & 100100 \\
\hline DMU 5 & Cabedelo & PB & 372,2 & 100251 & 100000 \\
\hline DMU 6 & Fortaleza & $\mathrm{CE}$ & 537,8 & 100803 & 100116 \\
\hline DMU 7 & Ilhéus & BA & 2730,56 & 100008 & 100000 \\
\hline DMU 8 & Imbituba & $\mathrm{SC}$ & 1932,13 & 100561 & 100025 \\
\hline DMU 9 & Itaguaí & $\mathrm{RJ}$ & 2211,68 & 104329 & 99773 \\
\hline DMU10 & Itajaí & SC & 514,49 & 100167 & 101018 \\
\hline DMU 11 & Itaqui & MA & 1473,48 & 107757 & 100200 \\
\hline DMU 12 & Maceió & AL & 569,37 & 101146 & 100014 \\
\hline DMU 13 & Manaus & AM & 270,2 & 101700 & 100334 \\
\hline DMU 14 & Natal & $\mathrm{RN}$ & 140,5 & 100063 & 100012 \\
\hline DMU 15 & Paranaguá & PR & 2178 & 102002 & 100865 \\
\hline DMU 16 & Pecém & CE & 2683,065 & 100000 & 100236 \\
\hline DMU 17 & Porto Alegre & RS & 100,2 & 102397 & 100034 \\
\hline DMU 18 & Recife & $\mathrm{PE}$ & 68,3 & 100852 & 100000 \\
\hline DMU 19 & Rio de Janeiro & $\mathrm{RJ}$ & 1893,84 & 104144 & 100590 \\
\hline DMU 20 & Rio Grande & RS & 1085,6 & 107279 & 101235 \\
\hline DMU 21 & Salvador & BA & 677,04 & 100182 & 100380 \\
\hline DMU 22 & Santarém & PA & 60,15 & 100064 & 100010 \\
\hline DMU 23 & Santos & SP & 8670,31 & 118147 & 106345 \\
\hline DMU 24 & São Francisco do Sul & $\mathrm{SC}$ & 1360,21 & 100412 & 100366 \\
\hline DMU 25 & São Sebastião & SP & 156,25 & 102053 & 100000 \\
\hline DMU 26 & Suape & $\mathrm{PE}$ & 2814,765 & 100608 & 100875 \\
\hline DMU 27 & Vila do Conde & PA & 348,2 & 105056 & 100059 \\
\hline DMU 28 & Vitória & ES & 2729,77 & 119256 & 100424 \\
\hline
\end{tabular}

\subsection{ESCOLHA DO MODELO DEA}

DEA Os modelos DEA “clássicos” CCR e BCC são os mais utilizados em modelagem. A escolha por um modelo CCR ou BCC traz, implicitamente, a seleção dos retornos constantes ou variáveis de escala, a geometria da superfície de envelopamento dos dados e as projeções de eficiência [13], [14].

A necessidade de realizar a translação dos dados dos outputs 1 e 2, conforme descritos na Tabela 3, implica a escolha do modelo BCC, por ser invariante a translações a outputs quando orientado a inputs e vice-versa. O modelo CCR não se adequa às translações, sendo assim desconsiderado para a aplicação ao problema de pesquisa.

Em relação à escolha da orientação do modelo BCC, cabe ressaltar o exposto acerca da atual conjuntura econômica do país. Julgou-se coerente orientar a modelagem BCC a input, pois permite que os investimentos possam ser reavaliados, com foco em economia de recursos em DMU ineficientes, sendo mantidas as projeções dos cenários de movimentação de cargas nos portos até 2023.

Além disso, o modelo BCC se aplica a escalas com retorno variável, o que se adequa ao problema de pesquisa. Segundo [7], o modelo BCC impõe uma fronteira de eficiência convexa, permite que DMU que operam com baixos valores de inputs tenham retornos crescentes de escala e as que operam com altos valores tenham retornos decrescentes de escala. A natureza dos portos, que operam em escalas diferentes de movimentação de cargas e envolvem montantes também diferenciados nos estudos do IPEA, também corroboram com a escolha do modelo BCC.

Os resultados do modelo BCC podem ser obtidos por técnica dos multiplicadores ou do envelope, também designadas como modelos primal e dual, respectivamente, produzindo os mesmos resultados para as eficiências. As equações do problema de programação linear ao modelo clássico BCC, no modo primal linearizado, orientado a input, correspondem à função objetivo (1) e às restrições (2), (3) e (4): 


$$
\begin{aligned}
& \text { MaxEffo }=\sum_{j=1}^{s} u_{j} y_{j o}+u_{*} \\
& \text { sujeito a } \\
& \sum_{i=1}^{r} v_{i} x_{i o}=1 \\
& -\sum_{i=1}^{r} v_{i} x_{i k}+\sum_{j=1}^{s} u_{j} y_{j k}+u_{*} \leq 0, \forall k \\
& v_{i}, u_{i} \geq 0, u_{*} \in R
\end{aligned}
$$

em que

$$
\begin{array}{ll}
\text { Effo: } & \text { eficiência da DMU } \\
\mathrm{V}_{\mathrm{i}}: & \text { peso (multiplicador) de input; } \\
\mathrm{u}_{\mathrm{j}}: & \text { peso (multiplicador) de output; } \\
\mathrm{X}_{\mathrm{i}}: & \text { input; } \\
\mathrm{y}_{\mathrm{j}}: & \text { output; e } \\
\mathrm{u}_{*}: & \text { fator de escala. }
\end{array}
$$

O Sistema Integrado de Apoio à Decisão (SIAD), desenvolvido por [32], foi utilizado como ferramenta de cálculo, conforme os dados da Tabela 3. O SIAD permitiu obter os resultados de eficiência das DMU para o modelo DEA-BCC, os pesos das variáveis e os alvos, que correspondem aos montantes de recursos a serem economizados nos projetos de cada porto, cujo conjunto de projetos foi modelado como ineficiente.

\section{ANÁLISE DOS RESULTADOS}

A fronteira de eficiência foi composta por nove portos: Antonina-PR, Aratu-BA, Belém-PA, Itajaí-SC, Porto Alegre-RS, Rio Grande-RS, Santos-SP, Vila do Conde-PA e Vitória-ES. A coluna "Alvo" indica a economia de recursos financeiros, planejados pelo IPEA para cada porto, em $\mathrm{R} \$$ milhões, de forma a transladar os portos para a fronteira de eficiência. Dessa forma, os portos considerados eficientes apresentam alvos nulos. A Tabela 4 apresenta os resultados da aplicação do modelo BCC, orientado a input, onde o resultado $100 \%$ indica as DMU que compõem a fronteira de eficiência.

Tabela 4: resultados

\begin{tabular}{clcccc}
\hline DMU & PORTO & UF & $\begin{array}{c}\text { INPUT } \\
\text { (R\$ milhões) }\end{array}$ & EFICIÊNCIA & $\begin{array}{c}\text { ALVO } \\
\text { (R\$ milhões) }\end{array}$ \\
\hline DMU 1 & Angra dos Reis & RJ & 255,2 & $12,9 \%$ & 32,89 \\
DMU 2 & Antonina & PR & 27,4 & $100,0 \%$ & 0 \\
DMU 3 & Aratu & BA & 659,59 & $100,0 \%$ & 0 \\
DMU 4 & Belém & PA & 35,2 & $100,0 \%$ & 0 \\
DMU 5 & Cabedelo & PB & 372,2 & $8,5 \%$ & 31,45 \\
DMU 6 & Fortaleza & CE & 537,8 & $11,4 \%$ & 61,09 \\
DMU 7 & Ilhéus & BA & 2730,56 & $1,0 \%$ & 27,4 \\
DMU 8 & Imbituba & SC & 1932,13 & $2,0 \%$ & 38,17 \\
DMU 9 & Itaguaí & RJ & 2211,68 & $12,7 \%$ & 280,39 \\
DMU 10 & Itajaí & SC & 514,49 & $100,0 \%$ & 0 \\
DMU 11 & Itaqui & MA & 1473,48 & $55,0 \%$ & 809,92 \\
DMU 12 & Maceió & AL & 569,37 & $10,2 \%$ & 57,94 \\
DMU 13 & Manaus & AM & 270,2 & $83,5 \%$ & 225,61
\end{tabular}




\begin{tabular}{clcccc}
\hline DMU & \multicolumn{1}{c}{ PORTO } & UF & $\begin{array}{c}\text { INPUT } \\
\text { (R\$ milhões) }\end{array}$ & EFICIÊNCIA & $\begin{array}{c}\text { ALVO } \\
\text { (R\$ milhões) }\end{array}$ \\
\hline DMU 14 & Natal & RN & 140,5 & $20,2 \%$ & 28,34 \\
DMU 15 & Paranaguá & PR & 2178 & $25,0 \%$ & 543,97 \\
DMU 16 & Pecém & CE & 2683,065 & $4,0 \%$ & 106,21 \\
DMU 17 & Porto Alegre & RS & 100,2 & $100,0 \%$ & 0 \\
DMU 18 & Recife & PE & 68,3 & $70,3 \%$ & 48 \\
DMU 19 & Rio de Janeiro & RJ & 1893,84 & $27,7 \%$ & 524 \\
DMU 20 & Rio Grande & RS & 1085,6 & $100,0 \%$ & 0 \\
DMU 21 & Salvador & BA & 677,04 & $26,8 \%$ & 181,39 \\
DMU 22 & Santarém & PA & 60,15 & $47,0 \%$ & 28,3 \\
DMU 23 & Santos & SP & 8670,31 & $100,0 \%$ & 0 \\
DMU 24 & São Francisco do Sul & SC & 1360,21 & $12,9 \%$ & 175,53 \\
DMU 25 & São Sebastião & SP & 156,25 & $56,7 \%$ & 88,58 \\
DMU 26 & Suape & PE & 2814,765 & $16,4 \%$ & 461,66 \\
DMU 27 & Vila do Conde & PA & 348,2 & $100,0 \%$ & 0 \\
DMU 28 & Vitória & ES & 2729,77 & $100,0 \%$ & 0 \\
\hline
\end{tabular}

A ineficiência calculada para 19 dos 28 portos sugere uma oportunidade para a reavaliação do montante de recursos financeiros destinados às obras de infraestrutura nos portos, desde que mantidos os cenários de aumento na movimentação de carga até 2023. Em termos práticos, os resultados ineficientes significam gastos elevados do input para o esperado retorno da produção dos outputs. Por outro lado, os nove portos eficientes apresentam volumes de investimentos compatíveis com o aumento da movimentação de cargas.

O modelo BCC é considerado "benevolente", em decorrência da "convexidade" aplicada à fronteira de eficiência. Essa característica garante que alguns portos com reduzidos volumes de investimentos sejam eficientes, como Antonina-PA (R \$ 27,4 milhões) e Belém-PA (R\$ 35,2 milhões), quando comparados a outros portos também eficientes, porém com investimentos mais vultosos, como Santos-SP (R\$ 8,67 bilhões) e Vitória-ES (R\$ 2,73 bilhões).

Por outro lado, a convexidade da fronteira de eficiência reduz consideravelmente os alvos para os portos com eficiência inferior a 10\%. Para o porto de Ilhéus-BA, que apresentou a menor eficiência (i.e. 1\%), bastaria a redução de $\mathrm{R}$ \$ 27,4 milhões do montante planejado em R\$ 2730,56 para atingir a fronteira de eficiência. Certamente o cálculo desse alvo em um modelo de retorno constante de escala (i.e. CCR) indicaria um valor superior, embora deva-se ressaltar o exposto sobre a inadequação do método CCR ao problema de pesquisa.

De qualquer forma, ínfimos índices de eficiência trazem à luz a necessidade de uma reavaliação dos gestores públicos e analistas dos órgãos e agências envolvidos, em relação aos investimentos em infraestrutura e as expectativas de retorno para os portos ineficientes. Em resumo, os resultados da modelagem DEA trazem questionamentos sobre o investimento de significativos recursos de infraestrutura para um conjunto ineficiente e apresenta o quanto em economia seria possível atingir em tais conjuntos. 


\section{CONCLUSÃO}

O transporte marítimo representa o principal modo de transporte ao comércio mundial e para o Brasil. Ao país, aproximadamente $95 \%$ de sua produção ao comércio exterior escoa a partir dos portos. Não obstante a relevância desse modo, o desempenho da atividade portuária apresenta resultados incompatíveis com a necessidade do país, conforme os dados expostos [2].

Diferentes programas voltados para a infraestrutura dos transportes em geral planejam a recuperação da infraestrutura aquaviária. Em paralelo, órgãos e agências especializadas estudam investimentos no setor e prospectam cenários de oferta e demanda que permitam assessorar gestores na tomada de decisão em investimentos. Nesse contexto, esta pesquisa apresentou os trabalhos do IPEA e da ANTAQ [3]-[6].

O Mapeamento IPEA apresentou investimentos em 265 projetos para modernizar a infraestrutura em 31 portos brasileiros, abrangendo a ampliação dos acessos por outros modais e obras em geral nos portos e nas áreas marítimas adjacentes. O PGO desenvolvido pela ANTAQ simulou cenários de movimentação de carga nos portos, com moldura temporal até o ano de 2023. Esses resultados foram utilizados como premissas desta pesquisa.

A modelagem DEA se mostrou adequada para a avaliação de investimentos em modernização dos portos, permitindo identificar o nível de eficiência dos diferentes projetos, localizar a ineficiência e propor metas tangíveis para a economia de recursos. Os investimentos planejados compuseram os recursos de entrada (inputs) e a capacidade ampliada dos portos os recursos de saída (output) da modelagem DEA.

Dadas as características do problema de pesquisa, julgou-se adequado aplicar o modelo BCC, tendo em vista a necessidade de translação dos dados dos outputs 1 e 2, devido à existência de valores não-positivos, além da variação em escala das DMU consideradas. A orientação do modelo a input permitiu identificar os montantes de recursos financeiros passíveis de uma redução, em decorrência da atual conjuntura econômica do país. Dos 28 portos incluídos nos estudos do IPEA e da ANTAQ, nove ocuparam a fronteira de eficiência. Aos 19 portos avaliados como ineficientes, foram calculados os alvos, indicando aos gestores metas tangíveis capazes de transladar os portos para a fronteira de eficiência.

\section{REFERÊNCIAS BIBLIOGRÁFICAS}

[1] IPEA, "Portos Brasileiros: Diagnóstico, Políticas e Perspectivas. Comunicados do IPEA Nr 48. Instituto de Política Econômica Aplicada. Série Eixos do Desenvolvimento Brasileiro,” Brasilia-DF, 2010.

[2] WEF, “The Global Competitiveness Report 2014-2015. Fórum Econômico Mundial,” 2015. [Online]. Available: http://reports.weforum.org/global-competitiveness-report-2014-2015. [Accessed: 17-Jun-2017].

[3] ANTAQ, "Subsídios técnicos para identificação de áreas destinadas à instalação de portos organizados ou autorização de Terminais de Uso Privativo em apoio ao Plano Geral de Outorgas. Anexos. Tomos 2.," Brasilia-DF, 2009.

[4] ANTAQ, "Subsídios técnicos para identificação de áreas destinadas à instalação de portos organizados ou autorização de Terminais de Uso Privativo em apoio ao Plano Geral de Outorgas. Relatório Final. Tomo 1," Brasilia-DF, 2009.

[5] C. A. da S. Campos Neto, B. Pêgo Filho, A. E. Romminger, I. M. Ferreira, and L. F. S. Vasconcelos, "Gargalos e demandas da infraestrutura portuária e os investimentos do PAC. Nota Técnica, n 2. Diretoria de Estudos Setoriais/IPEA,” Brasilia-DF, 2009.

[6] C. A. da S. Campos Neto, B. Pêgo Filho, A. E. Romminger, I. M. Ferreira, and L. F. S. Vasconcelos, "Gargalos e demandas da infraestrutura portuária e os investimentos do PAC: mapeamento Ipea de obras portuárias'. Texto para Discussão, n. 1423.," Instituto de Pesquisa Econômica Aplicada (Ipea), Brasilia-DF, 2009. 
[7] L. A. Meza, E. G. Gomes, and L. B. Neto, "Curso de análise de envoltória de dados," in XXXVII Simpósio Brasileiro de Pesquisa Operacional, 2005, pp. 20520-22547.

[8] J. Zhu, Quantitative models for performance evaluation and benchmarking: data envelopment analysis with spreadsheets, vol. 213. Springer, 2014.

[9] BRASIL, "Programa de Aceleração do Crescimento," 2015. [Online]. Available: http://www.pac.gov.br/transportes/portos. [Accessed: 17-May-2017].

[10] BRASIL, "Plano Nacional de Logística e Transportes (PNLT).” Ministério dosTransportes, Brasilia-DF, 2009.

[11] MPOG, "Plano Plurianual (PPA). Ministério do Planejamento Orçamento e Gestão.” Ministério do Planejamento Orçamento e Gestão, Brasilia-DF, 2008.

[12] D. Amora, "Dilma quer lançar PAC3 antes de terminar as obras do PAC 1," Folha de São Paulo, São Paulo, 19-May-2015.

[13] A. Charnes, W. W. Cooper, and E. Rhodes, "Measuring the efficiency of decision making units," Eur. J. Oper. Res., vol. 2, no. 6, pp. 429-444, 1978.

[14] R. D. Banker, A. Charnes, and W. W. Cooper, "Some models for estimating technical and scale inefficiencies in data envelopment analysis," Manage. Sci., vol. 30, no. 9, pp. 1078-1092, 1984.

[15] Y. S. Park, N. M. A. Mohamed Abdul Ghani, F. Gebremikael, and G. Egilmez, "Benchmarking environmental efficiency of ports using data mining and RDEA: the case of a US container ports," Int. J. Logist. Res. Appl., vol. 22, no. 2, pp. 172-187, 2019.

[16] B. Kalgora, S. Y. Goli, B. Damigou, H. T. Abdoulkarim, and K. K. Amponsem, "Measuring West-Africa Ports Efficiency Using Data Envelopment Analysis,” J. Transp. Technol., vol. 9, no. 3, pp. 287-308, 2019.

[17] D. M. E. de Paiva, M. A. V. Freitas, M. C. Barbosa, and N. D. Pizzolato, “Assessing the environmental management and operational efficiency of Brazilian public ports that export soybeans," Rev. Adm. Pública, vol. 53, no. 2, pp. 492-504, 2019.

[18] G. Dong, J. Zhu, J. Li, H. Wang, and Y. Gajpal, "Evaluating the Environmental Performance and Operational Efficiency of Container Ports: An Application to the Maritime Silk Road," Int. J. Environ. Res. Public Health, vol. 16, no. 12, p. 2226, 2019.

[19] S.-A. Julien, J. Cowie, and J. Monios, "Efficiency, productivity and returns to scale in ports: a comparison of data envelopment analysis and econometric estimation with application to Caribbean Small Island Developing States," Marit. Econ. Logist., pp. 1-26, 2018.

[20] I. C. Melo, P. N. A. Junior, A. E. Perico, M. G. S. Guzman, and D. A. do N. Rebelatto, "Benchmarking freight transportation corridors and routes with data envelopment analysis (DEA)," Benchmarking An Int. J., vol. 25 , no. 2 , pp. 713-742, 2018.

[21] C. Chen and J. S. L. Lam, "Sustainability and interactivity between cities and ports: a two-stage data envelopment analysis (DEA) approach,” Marit. Policy Manag., vol. 45, no. 7, pp. 944-961, 2018.

[22] B. Wiśnicki, L. Chybowski, and M. Czarnecki, "Analysis of the efficiency of port container terminals with the use of the data envelopment analysis method of relative productivity evaluation," Manag. Syst. Prod. Eng., vol. 25, no. 1, pp. 9-15, 2017.

[23] G. Cavone, M. Dotoli, N. Epicoco, and C. Seatzu, "Intermodal terminal planning by petri nets and data envelopment analysis," Control Eng. Pract., vol. 69, pp. 9-22, 2017.

[24] B. Wiegmans and P. Witte, "Efficiency of inland waterway container terminals: Stochastic frontier and data envelopment analysis to analyze the capacity design-and throughput efficiency," Transp. Res. Part A Policy Pract., vol. 106, pp. 12-21, 2017.

[25] W.-H. Huang, S.-L. Chao, and C.-C. Chang, "Assessment of differences in efficiency across strategic groups in the container shipping context: a data envelopment analysis," Int. J. Shipp. Transp. Logist., vol. 9, no. 6, pp. 651-672, 2017.

[26] M. G. M. Peixoto, M. C. A. Mendonça, M. A. Musetti, M. O. Batalha, and R. L. Sproesser, "Grain intermodal terminals: evaluation of pure technical efficiency by Data Envelopment Analysis," Production, vol. 27, 2017.

[27] D. Pjevcevic, M. Nikolic, N. Vidic, and K. Vukadinovic, "Data envelopment analysis of AGV fleet sizing at a port container terminal," Int. J. Prod. Res., vol. 55, no. 14, pp. 4021-4034, 2017. 
[28] N. Kutin, T. T. Nguyen, and T. Vallée, "Relative efficiencies of ASEAN container ports based on data envelopment analysis," Asian J. Shipp. Logist., vol. 33, no. 2, pp. 67-77, 2017.

[29] L. A. Meza, "Data envelopment analysis na determinação da eficiência dos programas de pós-graduação da COPPE/UFRJ," Universidade Federal do Rio de Janeiro (UFRJ), 1998.

[30] J. T. Pastor and J. L. Ruiz, "Variables with negative values in DEA," in Modeling data irregularities and structural complexities in data envelopment analysis, Springer, 2007, pp. 63-84.

[31] J. Sarkis, "Preparing your data for DEA," in Modeling data irregularities and structural complexities in data envelopment analysis, Springer, 2007, pp. 305-320.

[32] L. A. Meza, L. Biondi Neto, J. C. C. B. S. Mello, and E. G. Gomes, "ISYDS-Integrated System for Decision Support (SIAD-Sistema Integrado de Apoio à Decisão): a software package for data envelopment analysis model," Pesqui. Operacional, vol. 25, no. 3, pp. 493-503, 2005. 